cmp manuscript No.

(will be inserted by the editor)

\title{
Adiabatic Theorem without a Gap Condition
}

\author{
Joseph E. Avron, Alexander Elgart \\ Department of Physics, Technion, 32000 Haifa, Israel \\ e-mail: avron@physics.technion.ac.il \\ Received: 3 December 1998/ Accepted: 7 December 1998
}

\begin{abstract}
We prove the adiabatic theorem for quantum evolution without the traditional gap condition. All that this adiabatic theorem needs is a (piecewise) twice differentiable finite dimensional spectral projection. The result implies that the adiabatic theorem holds for the ground state of atoms in quantized radiation field. The general result we prove gives no information on the rate at which the adiabatic limit is approached. With additional spectral information one can also estimate this rate.
\end{abstract}

\section{Introduction and Motivation}

The adiabatic theorem of Quantum Mechanics describes the long time behavior of solutions of an initial value problem where the Hamiltonian generating the evolution depends slowly on time. The theorem relates these solutions to spectral information of the instantaneous Hamiltonian.

Traditionally, the adiabatic theorem is stated for Hamiltonians that have an eigenvalue which is separated by a gap from the rest of the spectrum. Folk wisdom is that some form of a gap condition is a sine qua non for an adiabatic theorem to hold. This is based on the following simple but at the same time rather forceful argument: The notion of Hamiltonian that depend slowly on time makes sense provided the system in question has a finite intrinsic time scale which determines what slow and fast mean. In quantum mechanics the intrinsic time scale is often determined by the gaps in the spectrum (and Planck's constant) [14]. For example, a Harmonic oscillator with natural frequency $\omega$, has gaps in the spectrum whose size is $\hbar \omega$. The condition for adiabaticity is $|\dot{\omega}|<<\omega^{2}$. In the $\omega \rightarrow 0$ limit the intrinsic time diverges and $\dot{\omega} \neq 0$ is never adiabatic. This suggests that one can not expect a general adiabatic theorem to hold in the absence of gaps. 
It is, of course, conceivable that in the absence of a gap some other property may determine a relevant and intrinsic time scale. For example, in the case of linearly crossing eigenvalues, the difference in slopes of the eigenvalues at the point of crossing $\alpha=\left(\dot{E}_{1}-\dot{E}_{2}\right)$, Fig. 1, determines a time scale, $\sqrt{\frac{1}{\alpha}}$, that takes over as the time scale associated with the gap diverges. An adiabatic theorem that builds on this fact goes back to Born and Fock [15]. But, at the same time, a general adiabatic theorem in the absence of a gap condition which does not use some other special properties, like a slope condition, seems unlikely and on physical grounds, morally wrong.

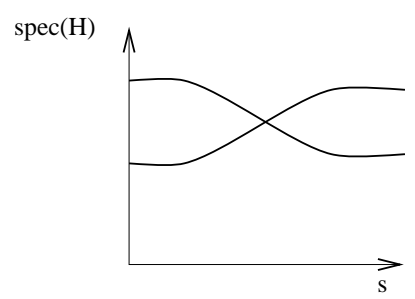

Fig. 1 Crossing Eigenvalues in Born Fock Theory

Nevertheless, the folk wisdom is actually wrong since we shall prove a general adiabatic theorem without a gap condition. All one really needs for the adiabatic theorem is a finite dimensional spectral projection for the Hamiltonian that depends smoothly on time. The role of the gap is to provide an a-priori rate at which the adiabatic limit is approached. In the absence of a gap, there is no such a-priori information on the rate at which the adiabatic limit is approached and it could be arbitrarily slow.

Our approach to an adiabatic theorem without a gap condition has some of the flavor of an operator analog of the Riemann-Lebesgue lemma [46]. If a function and also its derivative are in $L^{1}(\mathbb{R})$ then it is an elementary exercise that its Fourier transform decays at infinity at least as fast as an inverse power of the argument. The Riemann-Lebesgue lemma says that, in fact, the Fourier transform of any $L^{1}(\mathbb{R})$ function vanishes at infinity. The loss of a-priori information about the derivative translates to loss of information about the rate at which the function vanishes at infinity. In this analogy differentiability is the analog of the gap condition, and the $L^{1}(\mathbb{R})$ condition is the analog of the smoothness condition on the spectral projection.

A gap condition is associated with spectral stability. Situations without a gap condition often lead to spectral instabilities. This may suggest that an adiabatic theorem without a gap condition may be an academic exercise in the sense that it may have no applications and that its premise, the existence of a smooth spectral projection, is either contrived or would be 
hard to establish in applications. For example, for applications to atomic physics, where the essential spectrum is absolutely continuous, [18], embedded eigenvalues tend to dissolve to resonances [47] so it is unlikely that the projection associated to an embedded eigenvalue would be continuous. Indeed, we do not know of an application to embedded eigenvalues.

An interesting application of the adiabatic theorem without a gap condition is to eigenvalues at threshold. A ground state at threshold is a feature of any reasonable model Hamiltonian for atoms interacting with a radiation field. Models that do not have this property describe unstable atoms, or stable atoms in a world that has no soft photons. Models of atom-photon systems have the property that when the fine structure constant, $\alpha$, is small, the ground state describes the bound electrons of the atom and a photon field close to the vacuum. Soft photons are responsible for the absence of a gap in these models. A relatively simple yet interesting model for which the existence (and uniqueness) of the ground state [48, 1] as well as gaplessness [28] are known rigorously is the spin-boson Hamiltonian: The model of a two level system coupled to a radiation field. This has also been established for a model of non-relativistic QED [1, 6, 8, 8, 9]: A model of nonrelativistic electrons coupled to a radiation field with an ultraviolet cutoff. Unfortunately, for real QED [13, 16], where both the electrons and photons are treated as relativistic quantum fields, all that is rigorously known at present is on a perturbative level. Our original motivation was to prove an adiabatic theo-

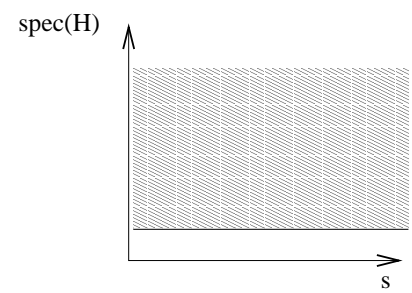

Fig. 2 An Eigenvalue at Threshold

rem for models describing atom-photon interaction. We proved this for the Dicke model, which is the simplest model of this kind, in [3]. We then realized that one could prove a much more general adiabatic theorem without a gap condition which is not specific to models of atoms in radiation field, but would cover these as a special case.

The adiabatic theorem without a gap condition resolves a problem regarding the relation between the quantum mechanics with and without radiation field. If the folk wisdom was true, and a gap condition was a necessary ingredient in the adiabatic theorem, one would expect the adiabatic theorem to hold for a two level system, but not for the spin-boson model. Since the 
spin-boson model is clearly a more accurate description of nature than the model of a two level system, the success of the adiabatic theorem in numerous applications where a two level model has been used, would appear like a mystery. The fact that adiabatic theorems do not really need a gap condition means that at least as far as the adiabatic theorem is concerned quantum mechanics without radiation and quantum mechanics with radiation sit in the same basket. An interesting problem that we do not resolve here is to show that not only is the adiabatic theory of quantum mechanics (without radiation) qualitatively correct, but it is also quantitatively accurate. For the Dicke model, some results in this direction are given in [3].

\section{Formulation of the Problem and the Main Result}

To formulate the problem of the adiabatic theory more precisely it is convenient, and traditional, to replace the physical time $t$ by the scaled time $s=t / \tau$. One is then concerned with the solution of the initial value problem

$$
i \dot{\psi}_{\tau}(s)=\tau H(s) \psi_{\tau}(s),
$$

in the limit of large $\tau . H(s)$ is a self-adjoint Hamiltonian which depends sufficiently smoothly on $s . \psi_{\tau}$ is a vector (in Hilbert space) valued function. We shall be more specific about what we mean by smoothness below. $H(s)$ evolves slowly in physical time for a long interval of time with finite variation in $H(s)$. Quantum adiabatic theorems say that the solution of the initial value problem is characterized, in the adiabatic limit $\tau \rightarrow \infty$, by spectral information. There is no single adiabatic theorem. Different adiabatic theorems focus on different aspects of the problem: What is assumed about $H(s)$ and $\dot{H}(s)$; about properties of the projection $P(s)$; the notion of smoothness, and what are the optimal error estimates, etc. All have the following structure: Let $P(s)$ be an appropriate family of spectral projections for $H(s)$. Let the initial data be such that $\psi_{\tau}(0) \in \operatorname{Range} P(0)$. Then, for an appropriate value of $\gamma \geq 0$,

$$
\operatorname{dist}\left(\psi_{\tau}(s), \text { RangeP }(s)\right) \leq O\left(\tau^{-\gamma}\right) .
$$

For $\gamma=0$ we take the right-hand side to mean $O\left(\tau^{-0}\right)=o(1)$.

In the present work we shall restrict ourselves to the case where $\dot{H}(s)$ is compactly supported. Then we can, without loss, take $s \in[0,1]$. Second, we shall restrict ourselves to uniform error estimates, i.e. error estimates that hold for all scaled time. This is actually the easier case. In adiabatic theory it is often possible to obtain much sharper results for times outside the support of $\dot{H}(s)$.

Our main result is the following:

Theorem 1 Suppose that $P(s)$ is smooth finite rank spectral projection, for the bounded, smooth Hamiltonian H(s). Then, the evolution of the initial 
state $\psi_{\tau}(0) \in$ Range $P(0)$, is such that dist $\left(\psi_{\tau}(s)\right.$, Range $\left.P(s)\right) \leq o(1)$ for all $s \in[0,1]$.

Remark 1 This is the weakest, but at the same time, the simplest, and most characteristic of our results. As it stands, it does not even apply to the Schrödinger operator because $H(s)$ is assumed to be bounded. In Sect. 5 we shall state a generalization of this result to unbounded operators. There are two reasons why we have chosen to state the weaker result. The first is that we did not want to obscure the central issue, and what is new in this work, behind a mask of technicalities. The second is almost ideological. The adiabatic problem is an infrared, low energy, problem. The central issue in an adiabatic theorem without a gap condition is to control low energy excitations. The unboundedness of Schrödinger operators is an ultraviolet problem. This problem has well developed analytical tools 32, 45, 50], and has nothing to do with the core of the infrared problem of adiabatic evolution. Once one has an adiabatic theorem without a gap condition for bounded operators, the extension to unbounded ones is technical.

Remark 2 We have stated the theorem with a condition of smoothness. Much less than smoothness is needed and we shall formulate a stronger result requiring only piecewise, twice differentiability of $P(s)$ in Sect. 5 . One reason why we have chosen to state a weaker result is again for simplicity, and the second is that it is likely that even the result in Sect. 5 is not optimal.

Remark 3 The theorem, as stated, does not cover the case of eigenvalue crossings. This is because at eigenvalue crossing the spectral projection $P(s)$ is not smooth $(\operatorname{Tr} P(s)$ is discontinuous). Eigenvalue crossings can be handled by a method due to Kato [33] and we shall state a stronger version of the theorem that allows for finitely many crossings in Sect. 5 .

\subsection{The Results of Davies and Spohn}

Davies and Spohn [19] studied the evolution of a driven, finite dimensional quantum system coupled to a heat bath. Their prime interest was the linear response of such a system which is closely related to the adiabatic limit. They choose a Hamiltonian of the form

$$
\tau\left(H_{q}(s)+H_{f}+\sqrt{\frac{1}{\tau}} H_{i}\right),
$$

where $H_{q}(s)$ is the time dependent Hamiltonian of the driven, finite dimensional, quantum sub-system, $H_{f}$ is the Hamiltonian of a quasi-free fermion field, and $H_{i}$ is the interaction. The coupling vanishes in the adiabatic limit 
$\tau \rightarrow \infty$. They show that the induced evolution of the finite dimensional sub-system is governed by a (finite dimensional) Hamiltonian of the form

$$
\tau H_{q}(s)+L(s) .
$$

Davies and Spohn then proceed to analyze the evolution of this finite dimensional system using some of the ideas that enter into the adiabatic theory of Kato [33]. Davies and Spohn do not prove an adiabatic theorem in the sense that the physical evolution adheres to a spectral subspace of the coupled Hamiltonian.

\section{A Panorama of Adiabatic Theorems}

In this section we recall some of the basic adiabatic theorems: Adiabatic theorems with a gap condition, for crossing eigenvalues, adiabatic theorems beyond all orders, and adiabatic theorems for scattering. We examine how these relate to the adiabatic theorem without a gap condition.

\subsection{Adiabatic Theorems with a Gap condition.}

The first satisfactory formulation and rigorous proof of an adiabatic theorem in the then new quantum mechanics was given in 1928 by Born and Fock [15. They were motivated by a point of view advocated by Ehrenfest [20], which identified classical adiabatic invariants as the observables that get quantized. The theorem they proved was geared to show that quantum numbers are preserved by adiabatic deformations.

Born and Fock proved an adiabatic theorem for Hamiltonian operators, $H(s)$, with simple discrete spectrum. They showed that in Eq. (2) one can take $\gamma \geq 1$. Their proof covers Hamiltonians like the one dimensional Harmonic oscillator, but not the Hydrogen atom, which has absolutely continuous spectrum at positive energies, and eigenvalues with multiplicities at negative energies.

In 1958 Kato [33] initiated a new strategy for proving adiabatic theorems. He introduced a notion of adiabatic evolution which is purely geometric. It is associated with a natural connection in the bundle of spectral subspaces. Kato's method was to compare the geometric evolution with the evolution generated by $H(s)$ and to show that in the adiabatic limit the two coincide. Using this idea, Kato was able to relax the condition that $H(s)$ had simple discrete spectrum. He showed that the adiabatic theorem holds when $P(s)$ is a finite dimensional spectral projection associated with an isolated eigenvalue. No assumption on the spectral type of $H(s)$ restricted to $R$ ange $P_{\perp}(s)$ need be made, Fig. 3.

Kato's results cover the case of Schrödinger operator for the Hydrogen atom. However it does not cover Schrödinger operators that arise for instance in the study of condensed matter physics, where there is no discrete 


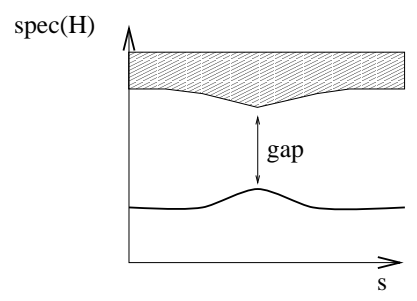

Fig. 3 Spectrum in Kato's Theory

spectrum at all. Kato's results were extended in [5,42], to $P(s)$ that need not be associated with an eigenvalue, and whose rank could also be infinite. In particular, the initial data could lie in a subspace corresponding to an energy band provided it is separated by a gap from the rest of the spectrum.

\subsection{Adiabatic Theorems beyond all orders.}

There are interesting and more delicate adiabatic theorems that apply provided one considers Eq. (2) for times $s$ that lie outside the support of $\dot{H}(s)$. Assuming a gap condition and smoothness (or analyticity) of $H(s)$ it has been shown [23, 24, 11, 35, 43] that the adiabatic theorem Eq. (2) holds with $\gamma=\infty$. Stronger results hold in the analytic case [39, 31, 29, 11].

\subsection{Adiabatic Theorems with Eigenvalue Crossings}

Born and Fock also studied the adiabatic theorem for crossing eigenvalues where the spectral projections have smooth continuations through the crossing point [15]. Born and Fock showed that if crossing is of order $m$ (linear crossing is $m=1)$ then Eq. (2) holds with $\gamma=1 /(m+1)$. This problem was later studied in much detail in [22] and [27].

Kato [33] also considered the adiabatic theorems for crossing eigenvalues. He did not make any explicit assumptions about how the eigenvalues behave near crossings. The only assumption he did make was that $P(s)$ could be continued through the crossings, and that there are finitely many crossings. Under these conditions he showed that Eq. (2) holds with $\gamma=0$.

\subsection{Adiabatic Theorems without a Gap Condition}

We are aware of one example of an adiabatic theorem without a gap condition for operators that have essential spectrum. This is a result of [4] for rank one perturbations of dense point spectra. The rate of approach to the adiabatic limit is $\gamma=1$ in Eq. (22). 


\subsection{Adiabatic Theorems for the Scattering Matrix}

Adiabatic scattering theory relates the time dependent scattering matrix to the time independent scattering matrix. Results in this direction are described in [41,39]. These have very little to do with the kind of adiabatic theorems we consider here. In scattering theory a time scale is determined by the initial data: The scattered particle spends a finite amount of time in the region of interaction, and in the limit that the interaction varies slowly, it does not see the variation in the Hamiltonian. The adiabatic theorems we are interested in consider a particle that spends a long time in the region of interaction.

\section{The Adiabatic Theorem and a Commutator Equation}

In this section we shall describe the proof of Theorem 1. To simplify the presentation, we shall stay away from making optimal assertions. In Sect. 5 we shall strengthen the result dropping most of the simplifying assumptions.

The center of this section, and the heart of the adiabatic theorem, is the commutator equation, Eq. (7). It is an operator valued equation for two bounded operators $X$ and $Y$. If one sets $Y=0$ one gets a commutator equation that goes back to Kato. The commutator equation with $Y=0$ has a bounded solution $X$ provided there is gap. If there is no gap the equation may, in some cases, have a bounded solution, but in general it will not. The basic idea behind the adiabatic theorem without a gap condition is that one can always solve this equation with $X$ bounded and $Y$ bounded and small. The smaller $Y$ the larger is the norm of $X$ in general, but this is all right, as we shall see.

In this section $H(s)$ is a family of bounded self-adjoint Hamiltonians that depends smoothly on $s$ so that $\dot{H}(s)$ is supported in the interval $[0,1]$. $H(s)$ generates unitary evolution as the solution of the initial value problem:

$$
i \dot{U}_{\tau}(s)=\tau H(s) U_{\tau}(s), \quad U_{\tau}(0)=1, \quad s \in[0,1] .
$$

We assume, without loss, that $H(s)$ has eigenvalue 0 and this eigenvalue has finite multiplicity. For this eigenvalue we formulate and prove our main result.

We recall the notion of adiabatic evolution 33, [. Let $U_{A}(s)$ be the solution of the initial value problem:

$$
i \dot{U}_{A}(s)=\tau\left(H(s)+\frac{i}{\tau}[\dot{P}(s), P(s)]\right) U_{A}(s), \quad U_{A}(0)=1, \quad s \in[0,1] .
$$

It is known that this unitary evolution has the intertwining property [5]:

$$
U_{A}(s) P(0)=P(s) U_{A}(s) .
$$


That is, $U_{A}(s)$ maps Range $P(0)$ onto Range $P(s)$. In particular, the solution of the initial value problem

$$
i \dot{\psi}(s)=\tau\left(H(s)+\frac{i}{\tau}[\dot{P}(s), P(s)]\right) \psi(s), \psi(0) \in \text { Range } P(0),
$$

has the property that $\psi(s) \in$ Range $P(s)$. We shall show that the Hamiltonian evolution, $U_{\tau}(s)$, is close to the adiabatic evolution $U_{A}(s)$.

We first formulate the basic lemma:

Lemma 1 Let $P(s), s \in[0,1]$, be a differentiable family of spectral projections for the self-adjoint Hamiltonian $H(s)$ with (operator) norm $\|\dot{P}(s)\|<$ $\infty$. Suppose that the commutator equation

$$
[\dot{P}(s), P(s)]=[H(s), X(s)]+Y(s)
$$

has operator valued solutions, $X(s)$ and $Y(s)$ with $X(s), \dot{X}(s)$ and $Y(s)$ bounded. Then

$$
\begin{gathered}
\left\|\left(U_{\tau}(s)-U_{A}(s)\right) P(0)\right\| \leq \\
\max _{s \in[0,1]}\left(\frac{2\|X(s) P(s)\|+\|(X(s) P(s)) P(s)\|}{\tau}+\|Y(s) P(s)\|\right) .
\end{gathered}
$$

The commutator equation, Eq. (7), can be viewed as a definition of $Y(s)$. The issue is not to find a solution to this equation, but rather to find solutions that make $Y$ small. In the case that there is a gap $\Delta$ separating the eigenvalue from the rest of the spectrum, a solution of the commutator equation is

$$
X(s)=\frac{1}{2 \pi i} \int_{\Gamma} R(z, s) \dot{P}(s) R(z, s) d z, \quad Y(s)=0 .
$$

Here $\Gamma$ is a circle in the complex plane, centered at the eigenvalue, and of radius $\Delta / 2$, Fig. 4. $R(z, s)=(H(s)-z)^{-1}$ is the resolvent at scaled time $s$. In this case the rate at which the adiabatic limit is obtained, is seen from Eq. (8) to be $1 / \tau$.

The strategy for proving the adiabatic theorem without a gap condition is to show that one can pick $Y$ so that its norm is arbitrarily small, possibly at the expense of large norm for $X$ and $\dot{X}$. So long as the norm of $X$ and $\dot{X}$ is finite, it can be compensated by taking $\tau$ large. This means that one can make the right-hand side of Eq. (8) arbitrarily small. The price paid is that there is, generally speaking, no information about the rate at which the adiabatic limit is obtained.

Proof Let $W(s)=U_{\tau}^{\dagger}(s) U_{A}(s)$ be the wave operator comparing the adiabatic and Hamiltonian evolution. Since

$$
\left\|U_{\tau}(s)-U_{A}(s)\right\|=\left\|U_{\tau}(s)(1-W(s))\right\|=\|1-W(s)\|,
$$




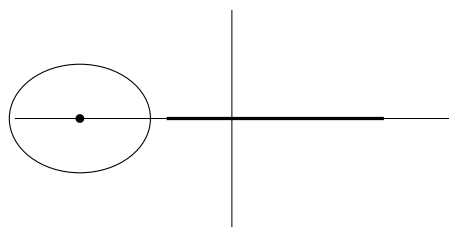

Fig. 4 A contour $\Gamma$ in the Complex Plane

we need to bound $W(s)-1$. From the definition of the adiabatic evolution, the commutator equation, and the equation of motion

$$
\begin{aligned}
\dot{W}(s)= & U_{\tau}^{\dagger}(s)([\dot{P}(s), P(s)]) U_{A}(s) \\
= & U_{\tau}^{\dagger}(s)([\dot{P}(s), P(s)]) U_{\tau}(s) W(s) \\
= & U_{\tau}^{\dagger}(s)([H(s), X(s)]+Y(s)) U_{\tau}(s) W(s) \\
= & -\frac{i}{\tau}\left(\dot{U}_{\tau}^{\dagger}(s) X(s) U_{\tau}(s)+U_{\tau}^{\dagger}(s) X(s) \dot{U}_{\tau}(s)\right) W(s) \\
+ & U_{\tau}^{\dagger}(s) Y(s) U_{A}(s) \\
= & -\frac{i}{\tau}\left(\left(U_{\tau}^{\dagger}(s) X(s) U_{\tau}(s)\right)-U_{\tau}^{\dagger}(s) \dot{X}(s) U_{\tau}(s)\right) W(s) \\
+ & U_{\tau}^{\dagger}(s) Y(s) U_{A}(s) \\
= & -\frac{i}{\tau}\left\{\left(U_{\tau}^{\dagger}(s) X(s) U_{\tau}(s) W(s)\right)-U_{\tau}^{\dagger}(s) X(s) U_{\tau}(s) \dot{W}_{(s)}(s)\right. \\
& \left.\quad-U_{\tau}^{\dagger}(s) \dot{X}(s) U_{\tau}(s) W(s)\right\}+U_{\tau}^{\dagger} Y(s) U_{A}(s) \\
& \quad-\frac{i}{\tau}\left\{\left(U_{\tau}^{\dagger}(s) X(s) U_{A}(s)\right)-U_{\tau}^{\dagger}(s) X(s)[\dot{P}(s), P(s)] U_{A}(s)\right. \\
& \left.-U_{\tau}^{\dagger}(s) \dot{X}(s) U_{A}(s)\right\}+U_{\tau}^{\dagger}(s) Y(s) U_{A}(s) .
\end{aligned}
$$

The lemma then follows by integration since $W(s)$ is unitary with $W(0)=1$.

Let us describe a solution of the commutator equation which is motivated by the solution Eq. (9) in the case of a gap. In order to have explicit error estimates and also in order to make the presentation simple and as elementary as possible, we choose a Gaussian regularizer. 
Definition 1 Let $g$ and e denote the Gaussian and Error functionฐ, and $\Phi$ be the special function defined below:

$$
g(\omega)=e^{-\pi \omega^{2}}, \quad e(\omega)=\int_{-\infty}^{\omega} d s g(s), \quad \Phi(\omega)=\theta(\omega)-e(\omega),
$$

$\theta$ is the usual step function which vanishes for negative argument. Also, let us denote the scaling of a function by

$$
g_{\Delta}(\omega)=g(\Delta \omega)
$$

and the multiplication operator by the argument by

$$
(\omega g)(\omega)=\omega g(\omega)
$$

An elementary lemma is:

Lemma $2 \Phi$ has finite $L^{1}$ norm and finite moments. In particular:

$$
\|\Phi\|_{1}=\frac{1}{\pi}, \quad\|\omega \Phi\|_{1}=\frac{1}{4 \pi} .
$$

Under scaling, $\Delta>0$ :

$$
\left\|\Phi_{\Delta}\right\|_{1}=\frac{1}{\pi \Delta}, \quad\left\|\omega \Phi_{\Delta}\right\|_{1}=\frac{1}{4 \pi \Delta^{2}} .
$$

We assume, without loss, that the spectral projection $P(s)$ is associated with the eigenvalue zero.

Lemma 3 Let $P(s)$ be a smooth spectral projection for $H(s)$ associated with the eigenvalue zero. Let $\Gamma$ be an infinitesimal contour around the origin in the complex plane. 2 Then the commutator equation has the solution

$$
\begin{aligned}
& X_{\Delta}(s)=A+A^{\dagger}, \quad A=P(s) \dot{P}(s) R(0, s)\left(1-g\left(\frac{H(s)}{\Delta}\right)\right) ; \\
& Y_{\Delta}(s)=-g\left(\frac{H(s)}{\Delta}\right) \dot{P}(s) P(s)+P(s) \dot{P}(s) g\left(\frac{H(s)}{\Delta}\right),
\end{aligned}
$$

where

$$
\begin{aligned}
\left\|X_{\Delta}(s) P(s)\right\| & \leq \frac{2\|\dot{P}(s) P(s)\|}{\Delta} \\
\left\|\left(X_{\Delta}(s) P(s)\right)\right\| & \leq \frac{2\left(\|\ddot{P}(s)\|+\| \dot{P}^{2}(s)\right) \|}{\Delta}+\frac{\pi\|\dot{P}(s)\|\|\dot{H}\|}{\Delta^{2}} .
\end{aligned}
$$

\footnotetext{
1 The error function we use differs by a factor and shift from canonical error function.

${ }^{2}$ The choice of Gaussian is not optimal. It would be more convenient to choose a regularizer which is a better approximant to a characteristic function and the reader may want to think of a Gaussian which is flattened at the top.
} 
Proof We start with a formal calculation. Let

$$
\begin{gathered}
F_{\Delta}(s)=g\left(\frac{H(s)}{\Delta}\right)-P(s) \\
X_{\Delta}(s)=\frac{1}{2 \pi i} \int_{\Gamma} d z\left(1-F_{\Delta}(s)\right) R(z, s) \dot{P}(s) R(z, s)\left(1-F_{\Delta}(s)\right) .
\end{gathered}
$$

Since $\dot{P}(s)=P(s) \dot{P}(s)+\dot{P}(s) P(s), X_{\Delta}(s)$ can be written as a sum of two adjoint terms, one of them is

$$
\begin{aligned}
\frac{1}{2 \pi i} & \int_{\Gamma} d z\left(1-F_{\Delta}(s)\right) R(z, s) P(s) \dot{P}(s) R(z, s)\left(1-F_{\Delta}(s)\right) \\
& =\frac{1}{2 \pi i}\left(1-F_{\Delta}(s)\right) P \dot{P}(s)\left(\int_{\Gamma} d z \frac{R(z, s)}{z}\right)\left(1-F_{\Delta}(s)\right) \\
& =P(s) \dot{P}(s) R(0, s)(1-P(s))\left(1-F_{\Delta}(s)\right) \\
& =P(s) \dot{P}(s) R(0, s)\left(1-P(s)-F_{\Delta}(s)\right) \\
& =P(s) \dot{P}(s) R(0, s)\left(1-g\left(\frac{H(s)}{\Delta}\right)\right)=A .
\end{aligned}
$$

We have used

$$
P(s) F_{\Delta}(s)=F_{\Delta}(s) P(s)=(g(0)-1) P(s)=0 .
$$

Using this integral representation of $X_{\Delta}(s)$ we now find $Y_{\Delta}(s)$. By our choice of $F_{\Delta}(s)$ we have $\left[F_{\Delta}(s), H(s)\right]=0$. Hence,

$$
\begin{aligned}
& {\left[X_{\Delta}(s), H(s)\right] } \\
= & \frac{1}{2 \pi i} \int_{\Gamma}\left[\left(1-F_{\Delta}(s)\right) R(z, s) \dot{P}(s) R(z, s)\left(1-F_{\Delta}(s)\right), H(s)-z\right] d z \\
= & \frac{1}{2 \pi i} \int_{\Gamma} d z\left(1-F_{\Delta}(s)\right)[R(z, s), \dot{P}(s)]\left(1-F_{\Delta}(s)\right) \\
= & \left(1-F_{\Delta}(s)\right)[P(s), \dot{P}(s)]\left(1-F_{\Delta}(s)\right) \\
= & {[P(s), \dot{P}(s)]-\left\{F_{\Delta}(s),[P(s), \dot{P}(s)]\right\}+F_{\Delta}(s)[P(s), \dot{P}(s)] F_{\Delta}(s) } \\
= & {[P(s), \dot{P}(s)]+F_{\Delta}(s) \dot{P}(s) P(s)-P(s) \dot{P}(s) F_{\Delta}(s) . }
\end{aligned}
$$

So a solution of the commutator equation is

$$
Y_{\Delta}(s)=-g\left(\frac{H(s)}{\Delta}\right) \dot{P}(s) P(s)+P(s) \dot{P}(s) g\left(\frac{H(s)}{\Delta}\right) .
$$

It remains to estimate the norms of $X$ and $\dot{X}$. Using the fact the Gaussian is its own Fourier transform,

$$
g\left(\frac{H(s)}{\Delta}\right)=\Delta \int_{\mathbb{R}} g(\Delta t) \exp [2 \pi i t H(s)] d t
$$


one checks that with our choice of $\Phi$

$$
R(0, s)\left(1-g\left(\frac{H(s)}{\Delta}\right)\right)=2 \pi i \int_{\mathbb{R}} \Phi(t \Delta) \exp [2 \pi i t H(s)] d t .
$$

Hence

$$
\left\|R(0, s)\left(1-g\left(\frac{H(s)}{\Delta}\right)\right)\right\| \leq 2 \pi\left\|\Phi_{\Delta}\right\|_{1}=\frac{2}{\Delta} .
$$

Using the equation for $X(s)$ this estimate proves the bound on $X(s)$. To get a bound on $\left(X_{\Delta}(s) P(s)\right)$, use the Duhammel formula,

$$
(\exp (2 \pi i t H(s)))=2 \pi i t \int_{0}^{1} d z e^{2 \pi i z t H(s)} \dot{H}(s) e^{2 \pi i(1-z) t H(s)} .
$$

Collecting the various terms give the claimed estimate.

As Lemma 3 shows, as $\Delta$ shrinks, the norms of $X(s)$ and $\dot{X}(s)$ may, and in general, will, grow. This, however is of no concern, as long as the norms remain finite, for one can always compensate for this growth by choosing $\tau$ large enough. The good thing about shrinking $\Delta$ is that this can be used to make the norm of $Y_{\Delta}$ small. Hence, we can always make the right-hand side of Eq. (8) arbitrarily small.

Lemma 4 Suppose that $H(s)$ is smooth with a zero eigenvalue with spectral projection $P(s)$ smooth and of finite rank. Let $g\left(\frac{H(s)}{\Delta}\right)$ be as above. Then $\left\|Y_{\Delta}(s) P(s)\right\|=\left\|g\left(\frac{H(s)}{\Delta}\right) \dot{P}(s) P(s)\right\| \rightarrow 0$ uniformly as $\Delta$ shrinks to zero.

Remark 4 We owe the proof below to Michael Aizenman.

Proof For the sake of simplicity suppose that $P(s)$ is a one-dimensional projection with $P(s) \psi(s)=\psi(s), \psi$ is normalized to 1 . Let $\varphi=\dot{P}(s) \psi$. Then, using $P(s) \varphi(s)=P(s) \dot{P}(s) \psi(s)=P(s) \dot{P}(s) P(s) \psi(s)=0$, we obtain

$$
\begin{aligned}
&\left\|g\left(\frac{H(s)}{\Delta}\right) \dot{P}(s) P(s)\right\|^{2}=\left\|g\left(\frac{H(s)}{\Delta}\right) \dot{P}(s) \psi(s)\right\|^{2}=\left\|g\left(\frac{H(s)}{\Delta}\right) \varphi(s)\right\|^{2}= \\
&\left\langle\varphi\left|g^{2}\left(\frac{H(s)}{\Delta}\right)\right| \varphi\right\rangle=\int_{\sigma(H(s))} g^{2}(x / \Delta) d \mu_{\varphi}(x)(29)
\end{aligned}
$$

where $\mu_{\varphi}$ denotes the spectral measure. Now, $g\left(\frac{x}{\Delta}\right)$ is bounded by one, and goes monotonically to zero for all $x \neq 0$, and $g(0)=1$. Hence

$$
\lim _{\Delta \rightarrow 0} \int_{\sigma(H(s))} g^{2}(x / \Delta) d \mu_{\varphi}(x)=\mu_{\varphi}(0)=0 .
$$

It follows that there is a sequence of $\Delta$ that makes $Y_{\Delta}(s)$ arbitrarily small. 
This completes the proof of Theorem 1 .

The physical interpretation of the adiabatic theorem without a gap condition is that although the adiabatic theorem "always" holds, it does so for different physical mechanisms. In the case that there is a gap in the spectrum the adiabatic theorem holds because the eigenstate is protected by a gap from tunneling out of the spectral subspace. In the case that there is no gap and the spectrum near the relevant eigenvalue is essential, the adiabatic theorem holds because essential spectrum is associated with states that are supported near spatial infinity. There is little tunneling to these states because of small overlap with the wave function corresponding to an eigenvalue which is supported away from infinity.

\section{Fine Print}

In this section we extend the adiabatic theorem without a gap condition to unbounded self-adjoint operators; replace the smoothness condition by a condition on differentiability and allow eigenvalue crossing. These extensions are technical in character and rely on existing machinery.

\subsection{Unbounded Hamiltonians}

The first, and perhaps the main, difficulty with unbounded operators $H(s)$ is the existence of solutions to the initial value problem, Eq. (1). For bounded operators the existence is a consequence of the Dyson formula, see e.g. Theorem X.59 in 45. For unbounded operators existence is more subtle so we chose a class for which this is the case:

Definition 2 A family of (possibly unbounded) self-adjoint Hamiltonians $H(s)$ is admissible if

1. $H(s)$ have the common domain in Hilbert space for all $s \in[0,1]$.

2. $H(s)$ is bounded from below by $\Lambda$.

3. $R(i, s)$ is bounded and differentiable and $H(s) \dot{R}(i, s)$ is bounded.

It is a consequence of our definition of admissibility that $A(t)=(H(t)-$ $\Lambda+1)$ is a strictly positive operator. Moreover, it is follows from property (11) by a closed graph theorem that $A(t) A(s)^{-1}$ is bounded. Since, for $t-s$ small, $\left\|(t-s)^{-1}\left(A(t) A(s)^{-1}-I\right)\right\|=\left\|A(s) \dot{A}(s)^{-1}\right\|+o(|t-s|)$, the last expression is bounded due to property (3). The existence of the unitary evolution for an admissible family of Hamiltonians follows now from (445], Theorem X.70):

Theorem 2 Let $X$ be a Banach space and let $I$ be an open interval in $\mathbb{R}$. For each $t \in I$, let $A(t)$ be the generator of a contraction semigroup on $X$ so that $0 \in \rho(A(t))$ and

1. The $A(t)$ have the common domain $D$. 
2. For each $\phi \in X,(t-s)^{-1}\left(A(t) A(s)^{-1}-I\right) \phi$ is uniformly strongly continuous and uniformly bounded in $s$ and $t$ for $t \neq s$ lying in any fixed compact subinterval of $I$.

3. For each $\phi \in X, C(t) \phi \equiv \lim _{t \rightarrow s}(t-s)^{-1}\left(A(t) A(s)^{-1}-I\right) \phi$ exists uniformly for $t$ in each compact subinterval and $C(t)$ is bounded and strongly continuous in $t$.

Then unitary evolution exists uniformly in s.

Then we can prove the following result.

Theorem 3 Suppose that $P(s)$ is finite rank spectral projection which is at least twice differentiable (as a bounded operator), for an admissible family $H(s)$. Then, the evolution of the initial state $\psi(0) \in$ Range $P(0)$, according to Eq. (1), is such that for all $s \in[0,1]$, dist $\left(\psi_{\tau}(s), \operatorname{RangeP}(s)\right) \leq o(1)$.

Proof Tracing the steps in Theorem 1 one sees that it is enough to check that the operators $X(s), \dot{X}(s)$ and $Y(s)$ are bounded uniformly in $s$. Now, by Eq. (17), $X_{\Delta}$ and $Y_{\Delta}$ are made of bounded operators such as $P, \dot{P}$ and $R$. Moreover, $X_{\Delta}$ is also differentiable as a bounded operator by our assumption that $P$ is twice differentiable, and by the admissibility condition that guarantees that $R$ is differentiable as a bounded operator. By the functional calculus $g(H(s))$ is also differentiable as a bounded operator. The only change is in the explicit estimate on the norm of $\dot{X}_{\Delta}$ in terms of $\Delta$, which is replaced by

$$
\begin{aligned}
\left\|\left(X_{\Delta}(s) P(s)\right)\right\| & \leq \frac{2\left(\|\ddot{P}(s)\|+\| \dot{P}^{2}(s)\right) \|}{\Delta} \\
& +\frac{\pi\|\dot{P}(s)(H(s)+i)\|\|R(s, i) \dot{H}\|}{\Delta^{2}},
\end{aligned}
$$

which is bounded for admissible $H(s)$.

\subsection{Piecewise Differentiability and Eigenvalue Crossing}

If at some time $0<s_{0}<1$ crossing of eigenvalues occurs, then the spectral projection associated with one of the eigenvalues, $P(s)$, is discontinuous at $s_{0}$ since its rank jumps. Suppose that $P(s), s \neq s_{0}$ is a spectral projection whose limit from the right and left coincide at $s_{0}$. In this case we can use an argument of Kato 33] that shows that global continuity together with piecewise smoothness is good enough.

Kato's argument goes as follows: Choose a small $\varepsilon$. The physical evolution follows the adiabatic evolution up to an arbitrarily small error on the interval $\left[0, s_{0}-\varepsilon\right]$. On the short interval $\left[s_{0}-\varepsilon, s_{0}+\varepsilon\right]$ the physical evolution is $\varepsilon$ takes Range $P\left(s_{0}-\varepsilon\right)$ close to itself. Since $P(s)$ is continuous at $s_{0}$, by assumption, this is equivalent to the statement that the physical evolution takes Range $P\left(s_{0}-\varepsilon\right)$ close to Range $P\left(s_{0}+\varepsilon\right)$, with an error that can be 
made arbitrarily small with $\varepsilon$. The physical evolution now follows the adiabatic evolution up to an arbitrarily small error on the interval $\left[s_{0}+\varepsilon, 1\right]$. Summarizing we have:

Theorem 4 Suppose that $P(s), s \neq s_{0} \in[0,1]$, is a finite rank spectral projection which is piecewise twice differentiable (as a bounded operator) and is everywhere continuous on $[0,1]$. Then the initial data $\psi_{\tau} \in \operatorname{RangeP}(0)$ evolve according to Eq. (1) so that dist $\left(\psi_{\tau}(s)\right.$, Range $\left.P(s)\right) \leq o(1)$ for all $s \in[0,1]$.

\section{The Rate of Approach to the Adiabatic Limit}

The general adiabatic theorems we have formulated give no information on the rate at which the adiabatic limit is approached. In fact, from the results of Born and Fock and Kato about eigenvalue crossings, it is clear that in the absence of a gap, the rate can be arbitrarily slow. To get interesting results on the rate at which the adiabatic limit is approached necessarily involves additional spectral information. In particular, if the bound state is either embedded or at the threshold of essential spectrum, with good behavior of the spectral measure at nearby energy, one expects to do better. An illustration of such estimates is given below.

Recall [36] that a (Borel) measure $\mu$ is called (uniformly) $\alpha$-Hölder continuous, $\alpha \in[0,1]$, if there is a constant $C$ such that for every interval $\Delta$

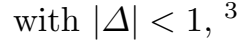

$$
\mu(\Delta)<C|\Delta|^{\alpha} .
$$

The interest in such measures comes from the fact [36, 17, 26] that they carry dynamical information and $\alpha$-continuous measures are the limits of $\alpha$-Hölder continuous measures. Knowing something about the Hausdorff dimension of the spectrum [36] then translates to information about the rate of approach of the adiabatic limit.

Corollary 1 If the spectral measure $\mu_{\varphi}(\Delta)$, is $\alpha$-Hölder continuous then the adiabatic limit is approached at least at rate $\gamma=\frac{\alpha}{2+\alpha}$. In the case of a family of Hamiltonians related by unitaries, $H(s)=V(s) H V^{\dagger}(s)$, with $\dot{V} V^{\dagger}$ bounded and differentiable the rate is at least $\gamma=\frac{\alpha}{1+\alpha}$.

Proof Let us note, first of all, that if the spectral measure $\mu_{\varphi}(\Delta)$, is $\alpha$-Hölder continuous then rhs of (30) is bounded by $\tilde{C}|\Delta|^{\alpha}$. Indeed,

$$
\begin{aligned}
\int_{\sigma(H(s))} g^{2}(x / \Delta) d \mu_{\varphi}(x) & <\sum_{n=0}^{\infty} g^{2}(n)\left(\int_{n \Delta}^{(n+1) \Delta} d \mu_{\varphi}(x)+\int_{-(n+1) \Delta}^{-n \Delta} d \mu_{\varphi}(x)\right) \\
& <C|\Delta|^{\alpha} \sum_{n=-\infty}^{\infty} g^{2}(n)=\tilde{C}|\Delta|^{\alpha} .
\end{aligned}
$$

\footnotetext{
${ }^{3}|\cdot|$ denote Lebesgue measure.
} 
Collecting the various error estimates one gets for the right hand side of Eq. (8) the upper bound

$$
\frac{A}{\Delta \tau}+\frac{B}{\Delta^{2} \tau}+\tilde{C}|\Delta|^{\alpha} .
$$

$A, B$ and $C$ are constants. For the case of the family of Hamiltonians related by unitaries, by Eq.(39) below, $B=0$. Optimizing the choice of $\Delta$ gives the result.

\subsection{Unitary Families}

By unitary families we mean the special case where the family $H(s)$ has the form

$$
H(s)=V(s) H V^{\dagger}(s)
$$

with $V(s)$ unitary. There are three points that we want to make about unitary families. The first is that such families are interesting in the context of adiabatic dynamics from the perspective of applications. The second is that there is some simplification that occurs for such families.

In a moving frame, the Schrödinger equation, Eq. (11), for $\psi_{\tau}=V \phi_{\tau}$ takes the form:

$$
i \dot{\phi}_{\tau}=\tau\left(H+\frac{i}{\tau} V^{\dagger}(s) \dot{V}(s)\right) \phi_{\tau}
$$

This leads to time independent Hamiltonian in the very special case: $V(s)=$ $e^{i s \sigma}$, with $\sigma$ self-adjoint (fixed) operator. The general case of unitary families, even in the rotating frame, leads to a time dependent problem, albeit one with a weak time dependent perturbation. As this perturbation is allowed to act for a long time, there is no obvious simplification in the rotating frame.

Unitary families often enter in applications. See for example, M. Berry's model of a spin half in a magnetic field 11]

$$
H(s)=B(s) \cdot \sigma,
$$

where $\sigma$ is a vector of Pauli matrices, and $B(s)$ a vector in $\mathbb{R}^{3}$ of unit length. This is a unitary family, which has all the intricacies of adiabatic theory associated with e.g. Zener tunneling [29].

Now we come to the simplification. In the case of unitary families one can improve the estimate of the norm of $\dot{X}$, which affects the estimate of the rate $\gamma$.

In the case of a unitary family

$$
P(s)=V(s) P V^{\dagger}(s), \quad \dot{P}(s)=\left[\dot{V}(s) V^{\dagger}(s), P(s)\right] .
$$


Hence, applying Eq. (37) to $X_{\Delta}(s)=A(s)+A^{\dagger}(s)$, where $A, A^{\dagger}$ are given by Eq. (17), we derive

$$
A(s)=V(s) P\left[V^{\dagger}(s) \dot{V}(s), P\right] R(0,0)\left(1-g\left(\frac{H}{\Delta}\right)\right) V^{\dagger}(s) .
$$

Therefore,

$$
\begin{aligned}
\dot{A}(s)=\left[\dot{V}(s) V^{\dagger}(s), A(s)\right]+V(s) P & {\left[V^{\dagger}(s) \dot{V}(s), P\right] R(0,0) . } \\
& \cdot\left(1-g\left(\frac{H}{\Delta}\right)\right) V^{\dagger}(s) .
\end{aligned}
$$

This identity is the reason why, for unitary families, one gets an improved rate with $\gamma=\frac{\alpha}{\alpha+1}$.

\subsection{Friedrichs Models}

Hölder continuity of the spectral measure gave an estimate of the rate $\gamma=\frac{\alpha}{\alpha+2} \leq \frac{1}{3}$, in the general case and $\gamma=\frac{\alpha}{\alpha+1} \leq \frac{1}{2}$ in the case of unitary families. Presumably, neither is optimal, since we used the additional spectral information only to estimate the norm of $Y_{\Delta}$, but not to improve the estimate on $X_{\Delta}$ and $\dot{X}_{\Delta}$. As a consequence, the best rate we get is $\gamma=\frac{1}{2}$. It is intriguing that for classical ergodic systems the approach to the adiabatic limit in the classical adiabatic theorem is with rate $\gamma=\frac{1}{2}$ 44]. This does not imply that the rate of approach to the adiabatic limit must be slow compared to the rate with a gap. In this subsection we shall consider a class of models, patterned after Friedrichs 21], where a more precise estimate of $\gamma$ can be made and where $\gamma$ can also take the value 1 in the absence of a gap.

Let us consider the family of unitarily related Hamiltonians $H(s)=$ $V(s) H V^{\dagger}(s)$. At any given time, $s$, there exists a representation of the Hilbert space such that $\mathcal{H}_{s}=\mathbb{C} \oplus L^{2}\left(\mathbb{R}^{d}, d \mu(k)\right)$ with inf $($ support $\mu)>-\infty$ and $\mu(0)=0$. A vector $\Psi \in \mathcal{H}$ is normalized by

$$
\Psi=\left(\begin{array}{c}
\omega \\
\psi(k)
\end{array}\right), \quad\|\Psi\|^{2}=|\omega|^{2}+\int_{\mathbb{R}^{d}}|\psi(k)|^{2} d \mu(k), \quad \omega \in \mathbb{C} .
$$

The (Friedrichs) Hamiltonian $H(s)$ in this representation acts on $\mathcal{H}_{s}$ like so:

$$
H(s) \Psi=\left(\begin{array}{cc}
0 & 0 \\
0 & k
\end{array}\right)\left(\begin{array}{c}
\omega \\
|\psi\rangle
\end{array}\right)=\left(\begin{array}{c}
0 \\
|k \psi\rangle
\end{array}\right) .
$$

The projection $P(s)$ has a form

$$
P(s)=\left(\begin{array}{ll}
1 & 0 \\
0 & 0
\end{array}\right),
$$


and the formal (reduced) resolvent $R(s)$ is given by

$$
R(s)=\left(\begin{array}{ll}
0 & 0 \\
0 & k^{-1}
\end{array}\right) .
$$

The time dependence of this unitary family can be encoded in the rate of change of two operators, namely

$$
\dot{P}(s)=\left(\begin{array}{ll}
0 & \left\langle f_{s}\right| \\
\left|f_{s}\right\rangle & 0
\end{array}\right)
$$

and

$$
(1-P(s))\left(\dot{V}(s) \dot{V}^{\dagger}(s)\right) P(s)=\left(\begin{array}{ll}
0 & 0 \\
\left|g_{s}\right\rangle & 0
\end{array}\right) .
$$

Suppose that $\dot{V}(s) V^{\dagger}(s)$ is bounded, has a bounded derivative, and

$$
\int_{B_{I}}\left|f_{s}\right|^{2} d \mu(k) \leq O\left(|I|^{2 \alpha}\right), \quad \int_{B_{I}}\left|g_{s}\right|^{2} d \mu(k) \leq O\left(|I|^{2 \alpha}\right), \quad \alpha \geq 0,
$$

where $B_{I}$ stands for a ball of radius $I$.

Proposition 1 For the Friedrichs model described above, the evolution of the state that starts as the bound state $\psi_{\tau}(0) \in$ Range $P(0)$, is such that it remains close to the instantaneous bound state and

$$
\operatorname{dist}\left(\psi_{\tau}(s), \text { Range } P(s)\right) \leq \begin{cases}O\left(\frac{1}{\tau}\right), & \alpha>1 ; \\ O\left(\frac{\log \tau}{\tau}\right), & \alpha=1 ; \\ O\left(\tau^{-\alpha}\right), & \alpha<1,\end{cases}
$$

for all $s \in[0,1]$.

Proof Formally

$$
X=R \dot{P}+\dot{P} R=\left(\begin{array}{lr}
0 & \left\langle\frac{f_{s}}{k}\right| \\
\left|\frac{f_{s}}{k}\right\rangle & 0
\end{array}\right)
$$

solves the commutator equation

$$
[X, H]=[P, \dot{P}] .
$$

Now choose

$$
X_{\epsilon}=R_{\epsilon} \dot{P}+\dot{P} R_{\epsilon}=\left(\begin{array}{lr}
0 & \left\langle\frac{f_{s} \chi(k>\epsilon)}{k}\right| \\
\left|\frac{f_{s} \chi(k>\epsilon)}{k}\right\rangle & 0
\end{array}\right),
$$

where $R_{\epsilon}=R(s) \chi(k>\epsilon)$ and pick $Y_{\epsilon}$ according to Eq. (7),

$$
\begin{aligned}
Y_{\epsilon} & =[P, \dot{P}]-\left[X_{\epsilon}, H\right] \\
& =\left(\begin{array}{lr}
0 & \left\langle f_{s} \chi(k<\epsilon)\right| \\
\left|f_{s} \chi(k<\epsilon)\right\rangle & 0
\end{array}\right) .
\end{aligned}
$$


Then

$$
\left\|Y_{\epsilon}\right\| \leq O\left(\int_{0}^{\epsilon}\left|f_{s}\right|^{2} d \mu(k)\right) \approx \epsilon^{2 \alpha}
$$

Since

$$
\int_{\mathbb{R}^{d} / B_{\epsilon}} \frac{|f|^{2}}{k^{2}} d \mu(k) \leq\left\{\begin{array}{l}
O\left(\epsilon^{2(\alpha-1)}\right) \text { if } \alpha \neq 1 \\
-O(\log \epsilon) \text { if } \alpha=1
\end{array}\right.
$$

we get the appropriate estimate of $\left\|X_{\epsilon}\right\|$. What remains is to estimate the norm of $\left(X_{\epsilon} \dot{P}(s)\right) P(s)$ :

$$
\begin{aligned}
\left(X_{\epsilon} \dot{P}(s)\right) P(s) & =R_{\epsilon} \ddot{P}(s) P(s)+\dot{R}_{\epsilon} \dot{P}(s) P(s) \\
& =B R_{\epsilon} \dot{P}(s) P(s)-R_{\epsilon} \dot{P}(s) B P(s)+R_{\epsilon} \dot{B} P(s) \\
& =B X_{\epsilon} P(s)-X_{\epsilon} B P(s)+R_{\epsilon}(1-P(s)) \dot{B} P(s),
\end{aligned}
$$

where $B=\dot{V}(s) V^{\dagger}(s)$. Making use of (46) we obtain that

$$
\left\|X_{\epsilon} P(s)\right\|,\left\|\dot{X}_{\epsilon} P(s)\right\| \leq\left\{\begin{array}{ll}
O\left(\epsilon^{2(\alpha-1)}\right) & \text { if } \alpha \neq 1 \\
-O(\log \epsilon) & \text { if } \alpha=1
\end{array} .\right.
$$

So, provided $\alpha>1$, we get the adiabatic theorem with $Y=0$ and with a rate $1 / \tau$. When $\alpha<1$ we optimize which gives

$$
\frac{\left\|X_{\epsilon} P(s)\right\|+\left\|\dot{X}_{\epsilon} P(s)\right\|}{\tau}+\left\|Y_{\epsilon}\right\| \leq\left\{\begin{array}{l}
O\left(\frac{\epsilon^{\alpha-1}}{\tau}\right)+O\left(\epsilon^{\alpha}\right) \text { if } \alpha<1 \\
-O\left(\frac{\log \epsilon}{\tau}\right)+O\left(\epsilon^{\alpha}\right) \text { if } \alpha=1
\end{array} .\right.
$$

Acknowledgements We are grateful to M. Aizenman for suggesting using the regularity of measures to streamline the proof of the main theorem, V. Bach, R. Seiler and H. Spohn for useful discussions and hospitality. This work was partially supported by a grant from the Israel Academy of Sciences, the Deutsche Forschungsgemeinschaft, and by the Fund for Promotion of Research at the Technion.

\section{References}

1. Arai, A., Hirokawa, M.: On the existence and uniqueness of ground states of a generalized spin-boson model. J. Funct. Anal. 151 (2), 455-503 (1997)

2. Arnold, V.I.: Geometrical Methods in the theory of Ordinary Differential Equations. Berlin-Heidelberg-New-York: Springer, 1983

3. Avron, J. E., Elgart, A.: An adiabatic theorem without a gap condition: Two level system coupled to quantized radiation field. Phys. Rev. A 58, 4300-4306 (1998)

4. Avron, J. E., Howland, J. S., Simon, B.: Adiabatic theorems for dense point spectra. Comm. in Math. Phys. 128, 497-507 (1990) 
5. Avron, J. E., Seiler, R., Yaffe, L. G.: Adiabatic theorems and applications to the quantum Hall effect. Comm. in Math. Phys. 110, 33-49 (1987), (Erratum: Comm. in Math. Phys. 153, 649-650 (1993))

6. Bach, V., Fröhlich, J., Sigal, I. M.: Mathematical theory of nonrelativistic matter and radiation. Lett. Math. Phys. 34, 183-201 (1995)

7. Bach, V., Fröhlich, J., Sigal, I. M.: Quantum electrodynamics of confined non-relativistic particles. Adv. in Math. 137, 299 (1998)

8. Bach, V., Fröhlich, J., Sigal, I. M.: Renormalization group analysis of spectral problems in quantum field theory. Adv. in Math. 137, 205-298 (1998)

9. Bach, V., Fröhlich J., Sigal, I. M., Sofer, A.: it Positive commutators and spectrum of non-relativistic QED. To appear

10. Berry, M.V.: Proc. Roy. Soc. Lond. A 392, 45 (1984); The quantum phase: Five years after. In: Geometric phases in physics. Shapere, A. and Wilczek, F., eds., Singapore: World Scientific, 1989

11. Berry, M.V.: Histories of adiabatic transition. Proc. Roy. Soc. Lond. A 429, 61-72 (1990)

12. Berry, M.V., Robbins, J.M.: Chaotic classical and half classical adiabatic reactions: Geometric magnetism and deterministic friction. Proc. Roy. Soc. Lond. A 442, 659-672 (1993). Proc. Roy. Soc. A 392, 45 (1984)

13. Bethe, H.A., Salpeter, E.E.: Quantum Mechanics of one and two electron atoms. New York: Plenum, 1977

14. Born, M.: The Mechanics of the Atom. New-York: Ungar, 1960

15. Born, M., Fock, V.: Beweis des Adiabatensatzes. Z. Phys. 51, 165-169 1928

16. Cohen-Tannoudji, C., Dupont-Roc, J., Grynberg G.: Atoms and Photons Interactions. New York: Wiley, 1992

17. Combes, J. M.: In: Differential equations with applications to mathematical physics. Boston: Academic Press 1993; Combes, J. M., Montcho, R.: Remarks on the relation between quantum dynamics and fractal spectra. J. Math. Anal. and Appl. 213, 698-722 1997

18. Cycon, H. L., Froese, R.G., Kirsch, W., Simon, B.: Schrödinger Operators. Berlin-Heidelberg-New-York: Springer 1987

19. Davies, E. B., Spohn, H.: Open Quantum Systems with Time-Dependent Hamiltonians and Their Linear Response. J. Stat. Phys. 19, 511-523 1978

20. Ehrenfest, P.: Adabatische Invarianten u. Quantentheorie. Ann. d. Phys. 51, 3271916

21. Friedrichs, K. O.: On the perturbation of continuous spectra. Comm. Pure Appl. Math. 1, 361-406 1948

22. Friedrichs, K. O.: Special topics in quantum theory. Lecture notes, Courant Institute of Mathematical Science, New York University, (1953); On the adiabatic theorem in quantum theory, Part I. Courant Institute of Mathematical Science, New York University, (1955); On the adiabatic theorem in quantum theory, Part II. Courant Institute of Mathematical Science, New York University, 1956

23. Garrido, L. M.: Generalized adiabatic invariance. J. Math. Phys. 5, 355-362 1964

24. Galindo, A., Pascual, P.: Quantum mechanics. Berlin-Heidelberg-NewYork: Springer-Verlag, 1991 
25. Golin, S., Knauf, A., Marmi, S.: The Hannay angles: Geometry, Adiabaticity and an example. Comm. in Math. Phys. 123, 95-122 1989

26. Guarneri, I.: On the dynamical meaning of spectral dimensions. Ann. Inst. H. Poincaré. To appear

27. Hagedorn, G.: Adiabatic Expansions near Eigenvalue Crossings. Ann. Phys. 196, 278-295 1989

28. Huebner, Spohn, H.: Ann. Inst. H. Poincaré Phys. Theor. 62, no. 3, 289 1995

29. Jakšić, V., Segert, J.: On the Landau Zener formula for two-level systems. J. Math. Phys. 34, 2807-2820 1993

30. Jarzinski, C.: Multiple-time-scale approach to ergodic adiabatic systems: Another look. Phys. Rev. Lett. 71, 8391993

31. Joye, A., Pfister, C.E.: Exponential Estimates in Adiabatic Quantum Evolution. Proceeding of the XII ICMP, Brisbane Australia (1997); Quantum Adiabatic Evolution. In On Three Levels. Fannes, M., Maes, C., Verbure, A., editors, London: Plenum, 1994

32. Kato, T.: Integration of the equation of evolution in a Banach space. J. Math. Soc. Japan. 5, 208-234 1953

33. Kato, T.: On the adiabatic theorem of quantum mechanics. Phys. Soc. Jap. 5, 435-439 1958

34. Kato, T.: Perturbation Theory for Linear Operators. Berlin-HeidelbergNew-York: Springer, 1966.

35. Klein, M., Seiler, R.: Power law corrections to the Kubo formula vanish in quantum Hall systems. Comm. in Math. Phys. 128, 1411990

36. Last, Y.: Quantum Dynamics and Decomposition of Singular Continuous Spectra. J. Funct. Anal. 142, 406-445 1996

37. Lennard, A.: Adiabatic Invariance to All Orders. Ann. Phys. 6, 261-276 1959

38. Lochak, P., Meunier, C.: Multiphase Averaging for Classical systems. Berlin-Heidelberg-New-York: Springer, 1988

39. Martinez, A.: Precise exponential estimates in adiabatic theory, J. Math. Phys. 35, 3889-3915 1994

40. Martinez, A., Nakamura, S.: Adiabatic limit and scattering. C.R. Acd. Sci. Paris. 318, 1153-1158 1994

41. Narnhofer, H., Thirring, W.: Adiabatic theorem in quantum statistical mechanics. Phys. Rev. A 26, 3646, 1982

42. Nenciu, G.: On the adiabatic theorem of quantum mechanics. J. Phys. A 13, L15-L18 1980

43. Nenciu, G.: Linear Adiabatic Theory: Exponential Estimates. Comm. in Math. Phys. 152, 479-496 1993

44. Ott, E.: Goodness of ergodic adiabatic invariants. Phys. Rev. Lett. 42, 1628-1631 1979; and Brown R., Ott, E., Grebogi, C.: Goodness of ergodic adiabatic invariants. J. Stat. Phys. 49, 511-550 1987

45. Reed, M., Simon, B.: Methods of Modern Mathematical Physics II. Fourier Analysis, Self-Adjointness. London: Academic Press, 1975

46. Riemann, B.: Ueber der Darstellbarkeit einer Function durch einen trigonometrishe Reihe. In: Math. Werke, Leipzig: Teubner, pp. 213-253 1876; Lebesgue, H.: Sur les Séries Trigonométriques. Ann. Sci. Ecole Norm. Sup. 20, 453-485 1903 
47. Simon, B.: The theory of resonances for dilation analytic potentials and the foundations of time-dependent perturbation theory. Ann. of Math. 97, 247-274 1973

48. Spohn, H.: Ground state(s) of the spin-boson Hamiltonian. Comm. Math. Phys. 123, 277-304 1989

49. Thouless, D.J.: Topological Quantum Numbers in Nonrelativistic Physics. Singapore: World Scientific, 1998

50. Yosida, K.: Functional Analysis. Berlin: Springer-Verlag, 1968

Communicated by B. Simon 